\title{
Lamb Wave Detection of Delaminations in Large Diameter Pipe Coatings
}

\author{
Jill Bingham and Mark Hinders*
}

\author{
Department of Applied Science, The College of William \& Mary in Virginia, NDE Lab @ 116 Jamestown Road, \\ Williamsburg, VA 23187-8795, USA
}

\begin{abstract}
This paper describes combined experiments and simulations to automate the extraction of ultrasonic guided wave mode arrivals in order to gain quantitative information about large-diameter pipeline coatings. The dynamic wavelet fingerprint technique (DWFT) is used to show differences between unknown coatings as well as to identify the presence of delamination and grinding flaws within the Lamb wave propagation path. Combined with complex multi-layered models to help interpret the guided wave feature changes, this extraction algorithm can be used for the detection of hidden flaws under a variety of protective coatings without having to disturb the coating or pipeline flow. High-resolution supercomputer simulations are developed using the elastodynamic finite integration technique (EFIT) accounting for the 3D interaction of realistic Lamb wave beams with finite-sized coating delaminations.
\end{abstract}

\section{INTRODUCTION}

The US Department of Transportation (DOT) installs and maintains over 2.3 million miles of pipeline nationwide that carry many substances including water, oil, natural gas and propane. Some of these pipelines have been in use for decades, but with optimum maintenance these pipelines could continue to function properly for years to come. One of the safeguards against damage to the pipelines are coatings, which protect them from the outside environment. Damage to these coatings could translate with time to irreversible pipeline damage, but due to the age of some of the pipelines the DOT doesn't have accurate records of the specific coatings on every pipeline installed across the country. Therefore, the DOT is interested in being able to access a small part of a pipe, nondestructively identify what type of coating is present on the surface, and also rapidly determine if it is in need of repair. Of particular interest are coating delaminations that could be a result of manufacturing flaws or the beginning signs of corrosion of the pipe substrate.

Here we describe the development of automatic guided wave interpretation for characterizing the type and bonding of protective coatings on large diameter pipelines. In these circumstances the signals received are too complex for interpretation without knowledge of the guided wave physics. We employ a signal processing technique called the Dynamic Wavelet Fingerprint Technique (DFWT) in order to render the guided wave mode information in twodimensional binary images. The use of wavelets allows us to keep track of both time and scale features from the original signals. With simple image processing we have developed automatic extraction algorithms for features that correspond

*Address correspondence to this author at the Department of Applied Science, The College of William \& Mary in Virginia, NDE Lab@116 Jamestown Road, Williamsburg, VA 23187-8795, USA;

E-mail: hinders@wm.edu to the arrival times of the guided wave modes of interest. Due to the dispersive nature of the guided wave modes, the mode arrival times give details of the compound structure in the propagation path.

For further understanding of how the guided wave modes propagate through the real structures, we have developed parallel processing, 3D elastic wave simulations using the finite integration technique (EFIT). This full field, numeric simulation technique easily examines models too complex for analytical solutions. We have developed the algorithm to handle built up 3D structures as well as layers with different material properties and surface detail. The simulations produce informative visualizations of the guided wave modes in the structures, but also directly mimic output from sensors placed in the simulation space for direct comparison to experiment. Using our previously developed mode extraction algorithms we were then able to compare our 3D EFIT data to their experimental counterparts with consistency.

\section{BACKGROUND}

Structural Health Monitoring (SHM) combines the use of attached sensors with artificial intelligence algorithms to automatically identify and monitor structural health issues. Ultrasonic guided wave methods [1-5] allow us to examine the interaction of multimode signals within key structural components. Since they propagate relatively long distances within pipe, plate and shell structures, guided waves allow inspection of greater areas with fewer sensors, making this technique attractive for a variety of applications $[6,7]$. This paper builds on a previous line of research we've developed over the past decade [8-23] Here, we will point out some of the major breakthroughs over the decades as well as recent advancements in the specific areas discussed in this work.

Elastic guided waves were first studied in 1885 by Lord Rayleigh [24] who was concerned with the propagation of 
surface waves along a solid, e.g. earthquakes. Twodimensional elastic waves in a bound plate are named for Lamb [25] who, in 1917, derived dispersion relations for the symmetric and antisymmetric modes. Mindlin subsequently presented a two dimensional theory of flexural waves of isotropic, elastic plates deduced from the three-dimensional equations of elasticity which were close to Lamb's original solutions, but computationally more simple [26]. In 1957 Worlton presented the first results from the experimental testing of aluminum plates in a tank for laminar flaws and radial cracks, and he also presented some suggestions of the practical use of Lamb waves [27]. His second experimental work with Lamb waves in 1961 rewrote the Lamb dispersion relations into the currently used notation, as well as graphically related the phase velocity of the modes to the frequency thickness of the plate [28].

Early work by Gazis in 1959 developed an exact elastic wave solution for a hollow cylinder [29, 30]. Since then many more authors have added to the guided waves in hollow cylinder literature. But it has also been proposed that the non-axisymmetric propagation of guided waves in a pipe correspond to Lamb waves in unwrapped pipes. Leonard and Hinders in 2005 showed that it is possible to obtain tomographic reconstructions of pipe wall thickness using mimicked crosshole geometries [10]. Li and Rose used this approximation in 2005 to derive a simplified model to calculate the phase velocities of non-axisymmetric flexural mode guided waves [31]. Luo, Zhao and Rose showed that although a pipe model is more accurate for a pipe experiment, a plate model often gives a quick and sufficient solution for a pipe [32].

By 1978, researchers had begun studying more complex plates and pipes, Nayfeh and Nassar for example found that the stiffer a bonding material the more influence it had on the wave propagation through laminated composites [33]. Claus and Kline analyzed the effect of interfacial surface roughness on the propagation of Stoneley waves guided along the interface of two dissimilar media [34]. They found that the Stoneley wave attenuation increased as a function of increasing surface roughness. Schoenberg studied the elastic wave behavior across slip boundaries where the displacement between two layers does not have to be continuous, and showed that the reflection and transmission coefficients are frequency dependent [35]. The experimental detection of slip boundaries was found possible by considering the changes in the reflection coefficients at an incident angle as a function of frequency [36]. Xu and Datta showed that increased stiffness and increased density have similar effects of lowering the cutoff frequency of guided wave modes in a bonded plate [37]. In 1991 Nayfeh presented an exact analytical treatment of elastic wave propagation in multi-layered anisotropic media using the transfer matrix method, which derives the individual layer solutions as wave amplitudes, then eliminating these amplitudes relates the stresses and displacements on one side of the layer to the other [38]. Laperre and Thys showed experimentally and theoretically that Lamb mode coupling between and polymer coating and an aluminum plate makes the bilayer system switch between modes [39]. In $1993 \mathrm{Xu}$ et al. used a method to decouple leaky Lamb wave data to determine the unknown elastic wave speed of a coating [40]. Rose et al. presented research on the correlation between the thickness of a coating of a bilayer system to the influence it has on the generation of Lamb wave modes [41]. The assertion was that if the layer thickness was $0.027<\mathrm{d}_{\mathrm{p}}<$ $0.083 \mathrm{~mm}$ the changes observed were minimal.

In 1995 Lowe wrote a review paper of the progression predictive methods for propagating waves in plates including dealing with multilayered plates, concentrating on the derivation of the 'Transfer Matrix' and 'Global Matrix' methods, and pointing out their short comings in the large frequency-thickness product [42]. Moulin et al. presented work starting in 2000 modeling the generation of Lamb waves using a coupled finite element-normal modes expansion method [43, 44]. In 2004 Duquenne et al. took this coupled method and extended it to the modeling of viscoelastic materials showing the attenuation of some modes in these layers [45]. By choosing modes with little attenuation from the viscoelastic layer, longer propagation distances are possible. Galan and Abascal then used a hybrid finite element-boundary element method, which explicitly included the attenuating characteristics of the coating to determine the Lamb mode conversion factors at defects under the coating [46]. Bashinger and Rose described how to use the global transfer matrix method to determine the dispersion equations, numerically solving for the phase velocity and attenuation of guided wave modes, and then verified these results with experiment [47]. In 2007 Luo and Rose applied phased array focusing to guided waves in a viscoelastic coated cylinder and showed that amplitude attenuation increased with the viscoelastic properties, but the phased array focusing increased the energy so that longer inspection distances could be achieved [48]. These results were obtained both numerically with a finite element model and experimentally.

Along with finite element techniques, numerical modeling using the elastodynamic finite integration technique (EFIT) has proven very useful for modeling guided wave behavior. Fellinger et al. originally developed the basic equations of EFIT along with a unique way to discretize the material parameters for insured continuity of stress and displacement across the staggered grid in 1995 [49]. Schubert et al. then adapted the EFIT equations into cylindrical coordinates (CEFIT) to investigate axisymmetric wave propagation in pipes with a 2D grid [50]. In 2001 Schubert presented results looking at elastic wave propagation in porous concrete but due to computational limitations could only model $5 \mathrm{~cm} \times 5 \mathrm{~cm} \times 10 \mathrm{~cm}$ spaces with periodic boundary conditions [51]. Then in 2004 Schubert gave an overview of the flexibility of EFIT with discretization in Cartesian, cylindrical and spherical coordinates and showed a wide range of modeling applications [52]. Rudd et al. then showed that the CEFIT could be extended to a 3DCEFIT to include bends in pipes and to model phased array focusing after pipe bends [53]. 3DCEFIT was implemented in parallel in order to be able to model complex pipes. 


\section{SIGNAL PROCESSING WITH WAVELET FINGERPRINTS}

In extended structures where the wavelength is on the same order as the thickness, the two boundaries cause multiple reflections and mode conversions, so that new wave packets develop that exist throughout the thickness. There are an infinite number of modes generated for higher frequency thickness products, although each of the modes, excluding the S0 and A0, has a cutoff frequency. At this cutoff frequency the phase velocity of the mode approaches infinity while the group velocity approaches zero.

The number of propagating modes is determined by the frequency thickness product and the way in which the waves are generated. The dispersive properties of the modes allow us to gain information about the structure as they propagate. With a known starting thickness we can choose an excitation frequency that generates a highly dispersive mode and then use this dispersive nature of the guided waves to interrogate structures for flaws.

Our philosophy is to excite complicated signals in order to keep all of the time series information, then post-process the waveforms to extract the most useful information about the modes of interest using the Dynamic Wavelet Fingerprinting Technique (DWFT). The DWFT implemented here relies on filtering the data with a discrete stationary wavelet (SWT) filter to remove a few layers of detail then passing the filtered signal through the fingerprinting algorithm.

Wavelets are very useful for analyzing time series data because the wavelet transform allows us to keep track of both time and frequency, or scale features. Whereas Fourier transforms break down a signal into a series of sines and cosines in order to identify the frequency content of the entire signal, wavelet transforms keep track of local frequency features in the time domain. Ultrasonic signal analysis with wavelet transforms was first studied by Abbate in 1994 who found that if the mother wavelet was well defined there was good peak detection even with large amounts of added white noise [54]. Massicotte, Goyette and Bose then found that even noisy EMAT sensor signals were resolvable using the multi-scale method of the wavelet transform [55]. One of the strengths compared to the fast Fourier transform was that the extraction algorithm did not need to include the inverse transform, the arrival time could be taken directly from the time frequency domain of the wavelet transform. In 2002 Perov et al. considered the basic principles of the formulation of the wavelet transform for the purpose of an ultrasonic flaw detector and concluded that any of the known systems of orthogonal wavelets are suitable for this purpose as long as the number of levels does not drop below 4-5 [56]. In 2003 Lou and Hu found that the wavelet transform was useful in suppressing non-stationary wideband noise from speech [57]. In a comparison study between the Wigner-Ville distribution and the wavelet transform, preformed by Zou and Chen, the wavelet transform out preformed the Wigner-Ville in terms of sensitivity to the change in stiffness of a cracked rotor [58]. In 2002 Hou and Hinders developed a multi-mode arrival time extraction tool that rendered the time series data in 2D time-scale binary images [59]. Since then this technique has been applied to multi-mode extraction of Lamb wave signals for tomographic reconstruction [12,60,61], time domain reflectometry signals wiring flaw detection $[62,63]$ and a periodontal probing device [64].

In general most of the information in a signal is contained in the approximations of the first few levels of the SWT. The details of these low levels often have mostly high frequency noise information. If we set the details of these first few levels to zero, when we reconstruct the signal with the inverse SWT we have effectively de-noised our signal to keep just information of the Lamb wave modes of interest. In our work, we start with the filtered ultrasonic signal and take a continuous wavelet transform. The CWT gives a surface of wavelet coefficients, this surface is then normalized between [0-1]. Then we perform a thick contour slice operation where the user defines the number of slices to use; the more slices, the thinner the contour slice. The contour slices are given the value of 0 or 1 in alternating fashion. They are then projected down to a $2 \mathrm{D}$ image where the result often looks remarkably like the ridges of a human fingerprint.

The problem has thus been transformed from 1dimensional signal identification problem to a $2 \mathrm{D}$ image recognition scenario. The power of the DWFT is that it reduces the time-series data into a binary matrix that is easily stored and transferred. There is also robustness to the algorithm, since different mother wavelets emphasize different features in the signals. For the most part in the research we've manually chosen the mother wavelet based on experience and using wavelets roughly shaped like the excitation pulses.

The last piece of the DWFT is the image recognition of the binary features that correspond to the modes of interest. We have found that different modes are represented in unique features in our applications. We've found that using a simple ridge counting algorithm on the $2 \mathrm{D}$ images is often a helpful way to identify some of the features of interest. Once a feature has been identified in the time scale space we have determined its arrival in the time domain as well and we can draw conclusions based on our knowledge of the guided wave theory.

\section{PIPELINE COATINGS}

For this research we concentrate on large diameter, lowpressure pipelines that are used for the transportation of the liquids and gases that fuel the nation and drive the economy. These types of pipelines are typically coated with epoxies and coal tar for protection. Large diameter pipes are awkward to work with, so we used a thick plate to mimic the guided waves in the pipe sample since pipe can be thought of as a plate rolled up on itself [32]. In a pipe the waveforms are somewhat more complicated because of the constructive and destructive interference that occurs as the helical waves wrap around the pipe. However, since we are dealing with large diameter pipes, we don't have to worry about the wrapping effects as much since the circumference is large enough that the modes separate out in time. Using a flat plate 
also allows us to easily apply coatings in a controlled manner in the laboratory.

Our goal was to identify different coating types, and determine delaminations of the coatings from the steel; we set up just this scenario. Using an $8^{\prime} \times 2^{\prime} \times 5 / 16^{\prime \prime}$ steel plate we covered four, 16" square areas with coatings. We obtained three different coatings: two epoxies (CANUSA HBE-95 from CCPC and Protal 7200 from Denso) and Bitumastic 50 from Carboline, which is a coal tar. In order to create a disbond under the coatings of uniform size and known location we taped down a double thickness of plastic to prevent the coatings from adhering to the steel. We used a test area that was a layer of Protal 7200 and a layer of Bitumastic 50 to mimic commonly used coating systems, in addition to the three individual coatings.

Table 1. Propagating Mode Group Velocities for a Steel Pipe $7.9375 \mathrm{~mm}$ Thick Excited with $500 \mathrm{kHz}$ Transducers and Arrival Times for Propagating $431.8 \mathrm{~mm}$

\begin{tabular}{|c|c|c|c|}
\hline & Mode & Group Velocity (mm/ $\mathbf{\mu s )}$ & Arrival \\
\cline { 2 - 4 } & S1 & 4.85 & 89.03 \\
\cline { 2 - 4 } Frequency: $500 \mathrm{kHz}$ \\
$\begin{array}{c}\text { Thickness: } 7.9375 \mathrm{~mm} \\
\text { Distance: } 431.8 \mathrm{~mm}\end{array}$ & A0 & 3.075 & 140.42 \\
\cline { 2 - 4 } & A1 & 2.9 & 148.89 \\
\cline { 2 - 4 } & S0 & 2.6 & 166.07 \\
\hline
\end{tabular}

The steel dispersion curve (Fig. 1) shows that guided waves excited in the pipe at $500 \mathrm{kHz}$ for a frequency thickness value of $3.96 \mathrm{MHz}-\mathrm{mm}$ have dispersive properties. At this frequency thickness product, four Lamb wave modes propagate with different velocities. The first arriving mode will be the S1 which will slow down with a thickness loss, next comes the A0 which is relatively non-dispersive in this region, followed by the $\mathrm{A} 1$ and $\mathrm{S} 0$ modes which speed up and slow down respectively with a thickness loss. Table $\mathbf{1}$ gives the group velocities of the modes as well as their arrival times for a propagation distance of $431.8 \mathrm{~mm}$.

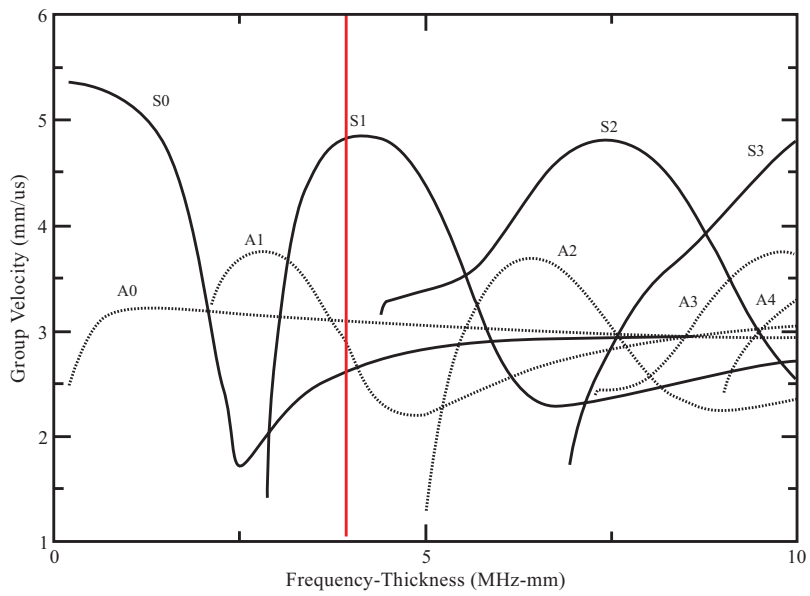

Fig. (1). Steel dispersion curves. $500 \mathrm{kHz}$ excitation gives a frequency-thickness product of $3.96 \mathrm{MHz}-\mathrm{mm}$ for a steel plate $5 / 16^{\prime \prime}$ thick.
Unfortunately this simple analysis only considers the steel pipe wall underneath the coatings that change the mode properties. Assuming that the coatings are linear viscoelastic layers, for the time harmonic case, elastic and viscoelastic solutions are identical with the exception that the viscoelastic material constants are complex and frequency dependent [47]. By inserting the real and complex material parameters for the layers into the model through the transfer matrix method [38], the global matrix method [42], or a hybrid finite element-normal modes expansion method [43, 45] we can obtain dispersion curves for the propagating Lamb wave modes. Through these methods it is apparent that there is the emergence of a decaying exponential term that causes attenuation of the propagating waves in the viscoelastic material due to the complex roots of the dispersion relations. We contend that the steel substrate is the largest factor in the propagation of the modes, and the effects of the coatings on these modes help us differentiate between the coatings. Due to the attenuation caused by the viscoelastic layers we expect a damping of the guided modes compared to the clean steel plate. In the local frequency thickness region we also expect the dispersive nature of the pipe and layer system to be similar to that of the pipe. We expect this to hold truer for a layer that is more elastic rather than viscoelastic. The stiffer the layer, the more effect it has on the guided elastic modes in the underlying substrate [33]. The coal tar is a soft sticky material that indents with fingerprints when touched. On the other hand, the epoxies are stiff hardened layers that cannot easily be cut with a utility knife. From product data sheets we've determined the hardness and specific gravity, $S_{g}$, of the different materials found in Table 2. The three different hardness scales are necessary because of the viscoelastic differences between the coatings and the steel. Shore A is the lowest scale, followed by Shore D for the viscoelastic properties then finally the Vickers scale tests the elastic properties of the steel with a $5 \mathrm{~kg}$ weight. This shows a great mismatch in hardness between the Bitumastic 50 and the steel pipe, while the two epoxies have comparable albeit lower hardness than the steel. This means that we would expect that the guided waves would show more dispersive traits in the samples covered with the epoxy coatings than the coal car.

Table 2. Hardness and Specific Gravities for the Coatings and Steel for the Pipeline Tests. Due to the Wide Range of the Viscoelastic Coatings and Elastic Steel, Three Different Hardness Scales are Used: Shore A, Shore $D$ and the Vickers

\begin{tabular}{|l|c|c|c|}
\hline \multicolumn{1}{|c|}{ Material } & Hardness & Hardness Unit & $\mathbf{S}_{\mathbf{g}}$ \\
\hline \hline CANUSA HBE-95 & $>85$ & Shore D & 1.031 \\
\hline Denso Protal 7200 & $85 \pm 2$ & Shore D & 1.53 \\
\hline Carboline Bitumastic 50 & 15 & Shore A & $1.50[48]$ \\
\hline Steel & 120 & HV5 & 7.8 \\
\hline
\end{tabular}

For the experimental tests of each of the coated areas on the large steel plate, we used linear stepper motors to take controlled steps down the sides of each coated test area on 
the large steel plate. Fig. (2) shows the configuration of each of the coating squares. The transmitting transducer (left) and the receiving transducer (right) were stepped in parallel down the sides of the square taking a waveform every $2 \mathrm{~mm}$ for 72 steps. This can be thought of stepping around the circumference of a real pipe sample, or using a single transmit-receive transducer with the guided waves propagating all the way around the circumference. Fig. (3) shows sample waveforms from three of the coating configurations: the HBE-95, Bitumastic 50 and Protal 7200 + Bitumastic 50. These waveforms were taken from flawless sections of the samples. Fortunately we immediately see some differences between the three different waveforms. The raw waveforms from the tests with epoxy coatings have lower signal to noise ratios, this dampening was expected due to the viscoelastic properties of the coatings attenuating the signals some.

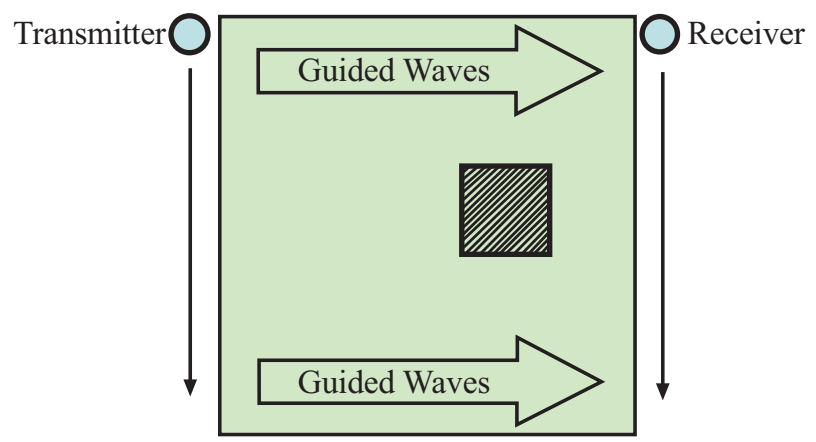

Fig. (2). Coating test configuration. The transmitting transducer (left) and receiver (right) were stepped $2 \mathrm{~mm}$ at a time down the sides of the coating square (shown in green) pulsing at $500 \mathrm{kHz}$ in order to excite the guided Lamb waves at each step. Each coating has a manufactured delamination of finite size (2" x 2 ") in the area indicated.

Using a SWT to remove three levels of details before applying the DWFT with the 'gaus2' mother wavelet we obtain thumbprints containing a triangular feature for each of the coatings (Fig. 4). The thumbprints show some interesting features. The Bitumastic 50 feature is very clean without any other mode features present, while the two epoxy samples indicate the presence of other modes. Of the three, the Bitumastic 50 is the first arriving, followed by the HBE-95 and finally the Protal $7200+$ Bitumastic 50. Drawing from Table 1 we suspect that this mode feature corresponds to the S0 guided wave mode. As expected, the arrival times for the epoxy coated samples are delayed when compared to the pipe wall alone. The earlier arriving guided wave modes seem to be very attenuated in the coatings. The steel dispersion curves indicate a slowing of the S0 mode, this would correspond to the triangular feature moving to the right. The material property mismatch between the steel and the Bitumastic 50 seems great enough that the elastic waves are not very affected by this layer, staying primarily in the steel pipe wall.

As the transducers are scanned down the sides of the coating samples, waveforms are collected from paths that do not propagate through the delamination before and afterwards as well as those on the edges of the flawed area and passing directly through the delamination. Fig. (5) shows a sequence of thumbprints from the 72 step scan of the HBE95 coated sample. As the propagation path begins to interact with the delamination the triangular feature moves to the right as expected. This signifies a slowing of the mode. When passing directly through the delamination the amplitude of the signal significantly drops as well as having a later arrival at $276.8 \mu \mathrm{s}$. This is a drastic slowing of the guided wave mode, indicating a possible mode conversion when propagating directly through the delamination. The propagation paths that interact with the edges of the flaw seem to more closely follow what we would expect from a thickness loss. For steel this $2 \mathrm{~mm}$ thickness loss would be shown with a $28.8 \mu$ s delay of the arrival of the S0 mode, here we see the arrival $49 \mu$ s later at $228.9 \mu$ s. The dispersion of the epoxy-steel system is intensified.

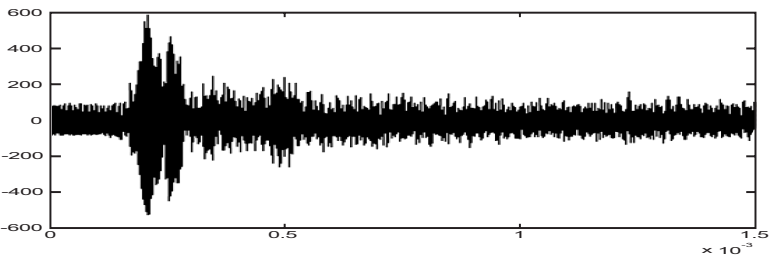

(a) HBE - 95

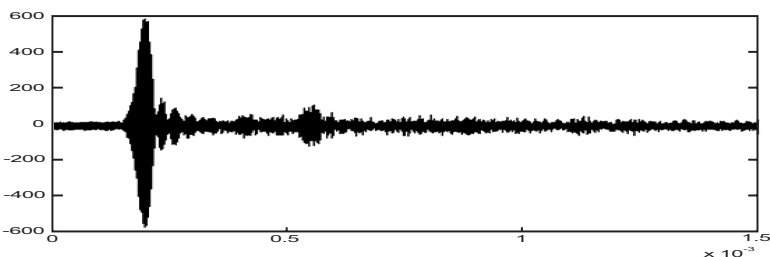

(b) Bitumastic 50

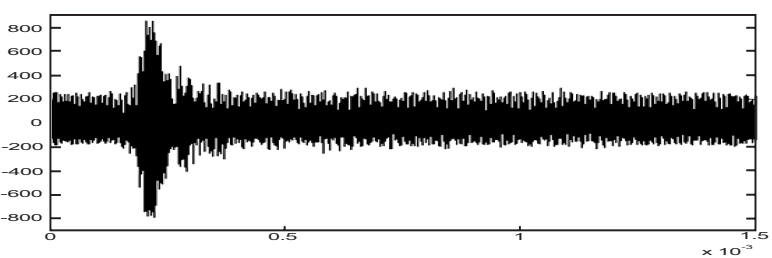

(c) Protal $7200+$ Bitumastic 50

Fig. (3). Three sample waveforms from three different coatings. (Top) HBE-95 (Middle) Bitumastic 50 (Bottom) Protal $7200+$ Bitumastic 50.

If we consider the same sort of progression for the Bitumastic 50 sample we see that the delamination has little effect of the arrival time of the S0 mode, but instead the delamination lessens the damping of the other modes for a portion of their propagation so we see their presence (Fig. 6). With the guided wave energy mostly contained in the pipe wall, it makes sense that there would not be a dispersion effect in this sample. With a delamination the pipe wall thickness does not change. Taken to an extreme, this material property mismatch can be compared to the interface between steel and water. When submerged, guided waves still propagate through the steel wall with little leakage into the surrounding liquid. Here there is more energy attenuated into the coal tar layer because of its viscoelastic properties. Interestingly, the amplitude of the S0 mode decreases when propagating directly through the delamination as indicated in Fig. (6c) by the fewer ridges in the triangular feature. 


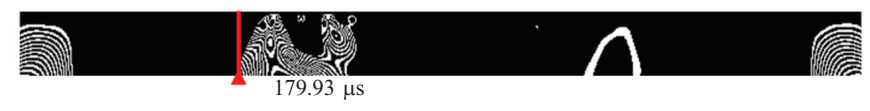

(a) HBE - 95

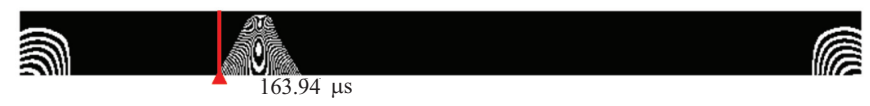

(b) Bitumastic 50

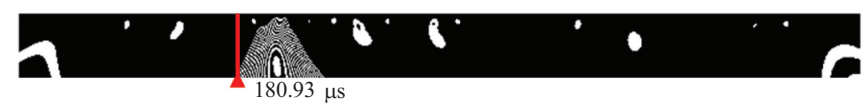

(c) Protal $7200+$ Bitumastic 50

Fig. (4). Three sample thumbprints from regions of the plate with three different coatings. (Top) HBE-95 (Middle) Bitumastic 50 (Bottom) Protal 7200 + Bitumastic 50 .

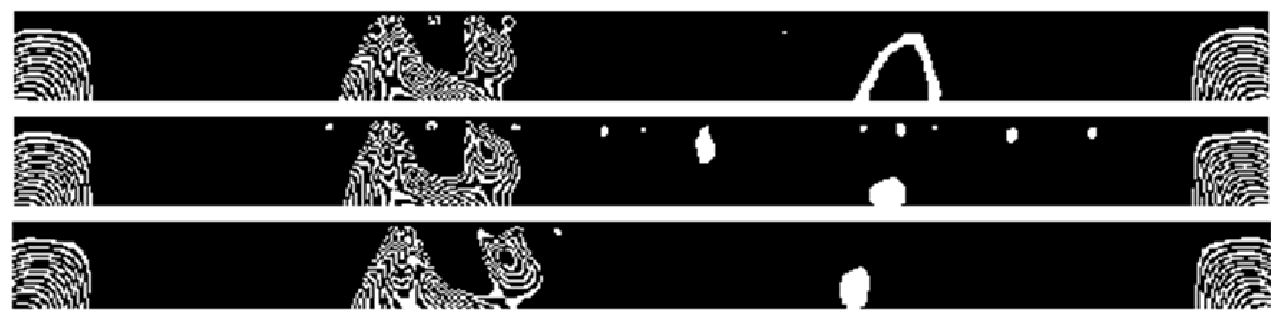

(a) Before flaw

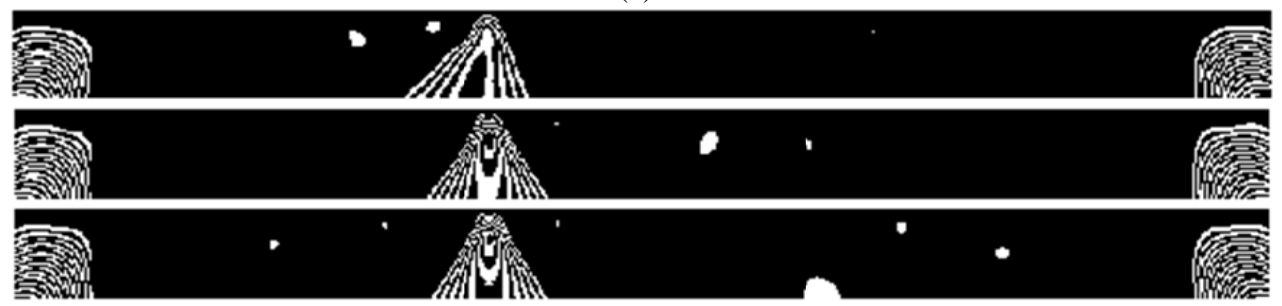

(b) Edge entering flaw

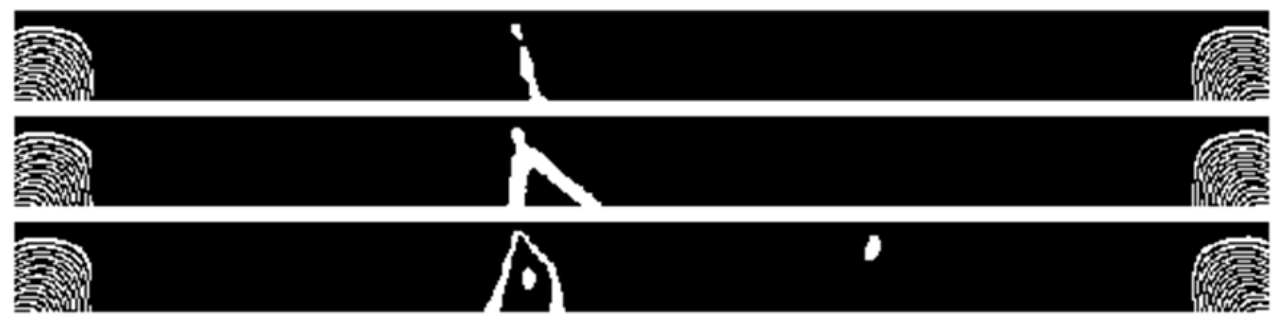

(c) Delamination

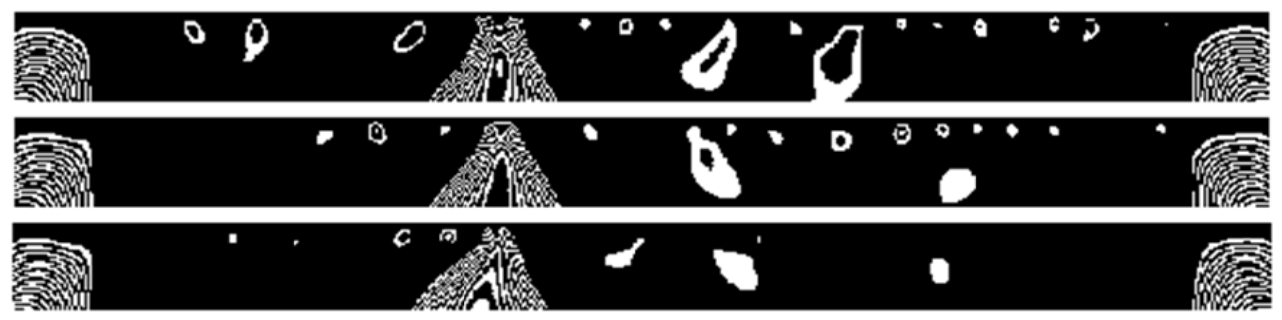

(d) Edge exiting flaw

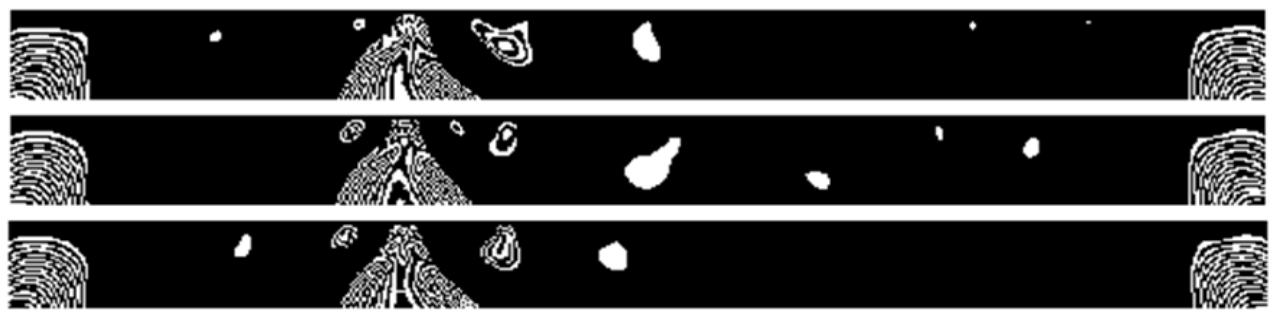

(e) After flaw

Fig. (5). Thumbprints for HBE-95 scan past delamination section. 


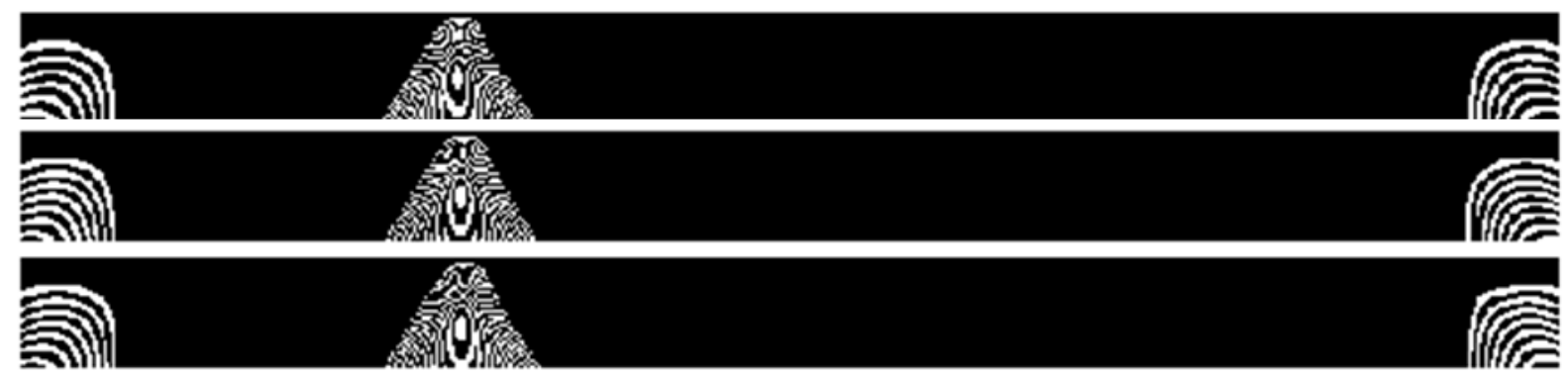

(a) Before flaw

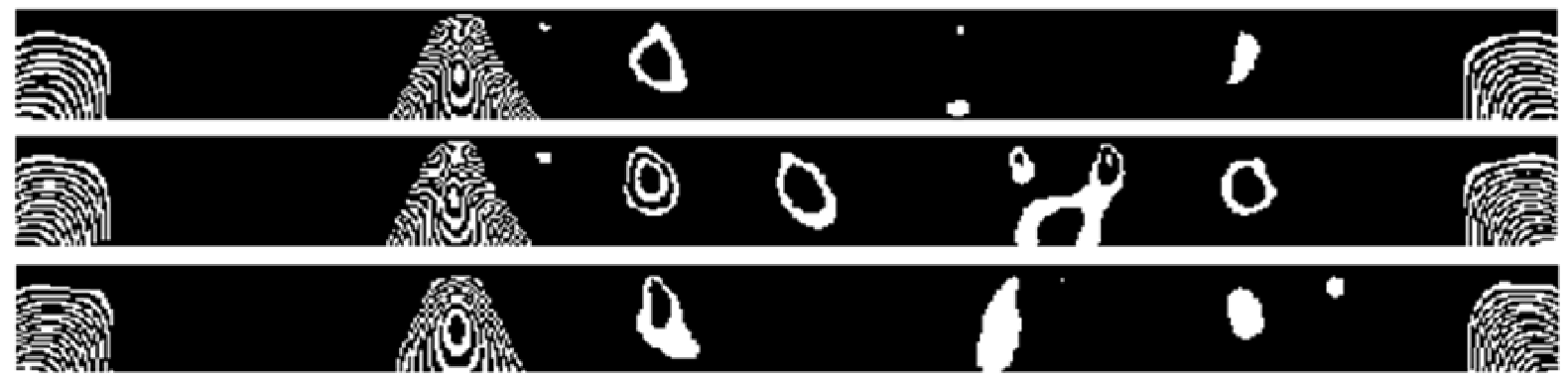

(b) Edge entering flaw

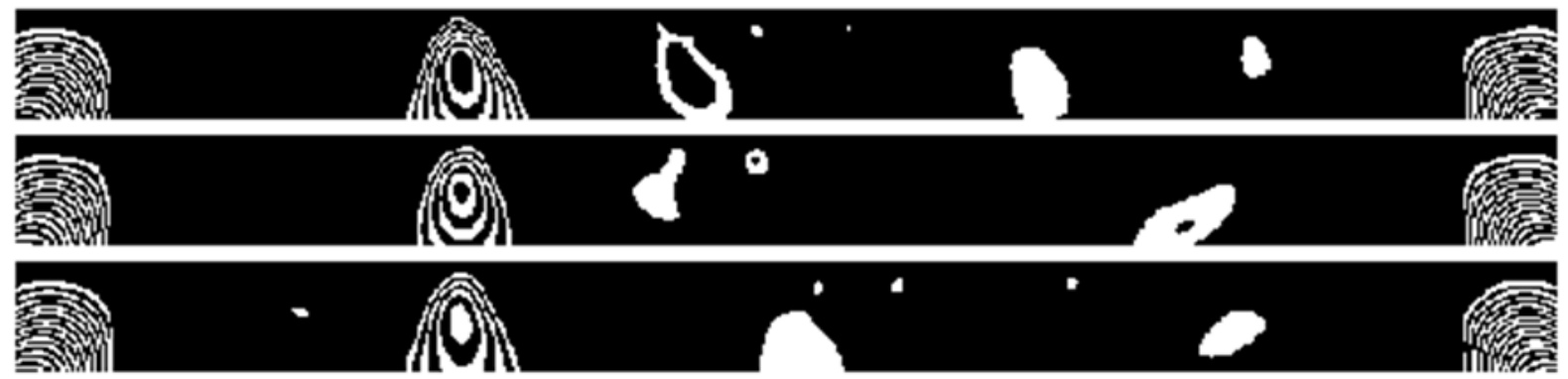

(c) Delamination

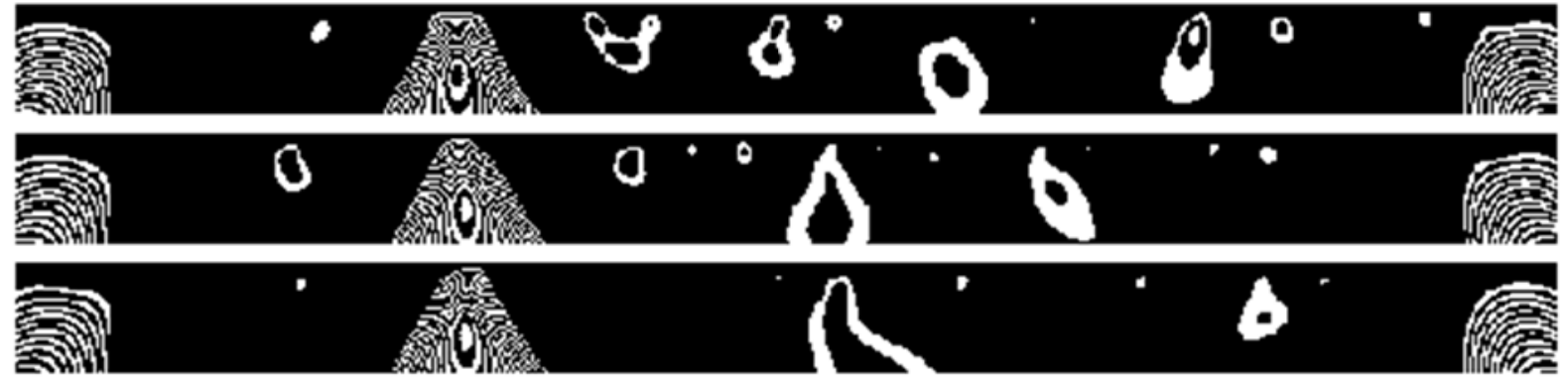

(d) Edge exiting flaw

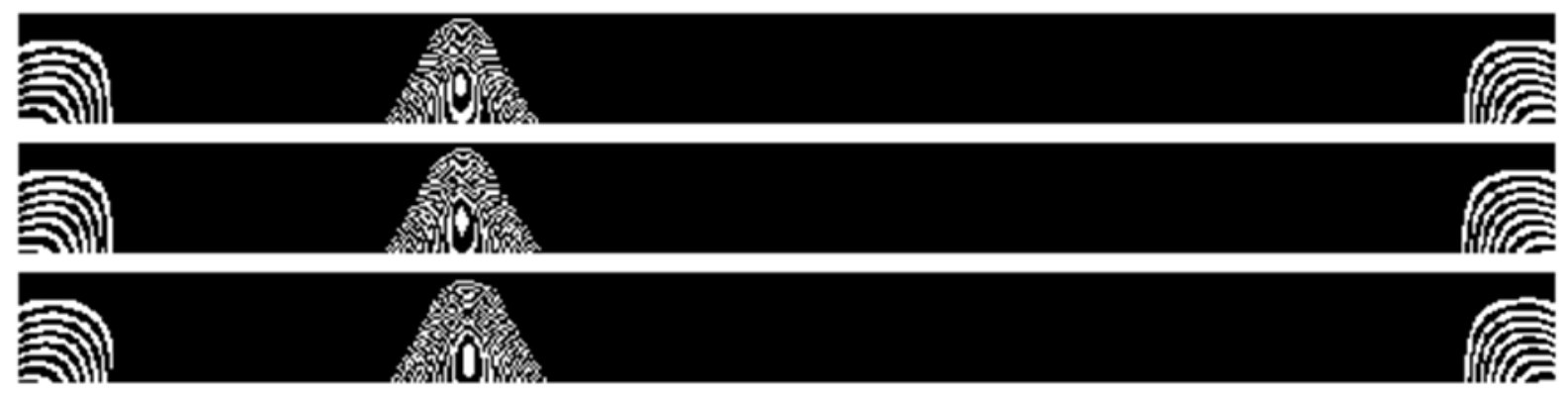

Fig. (6). Thumbprints for Bitumastic 50 scan past delamination section. 


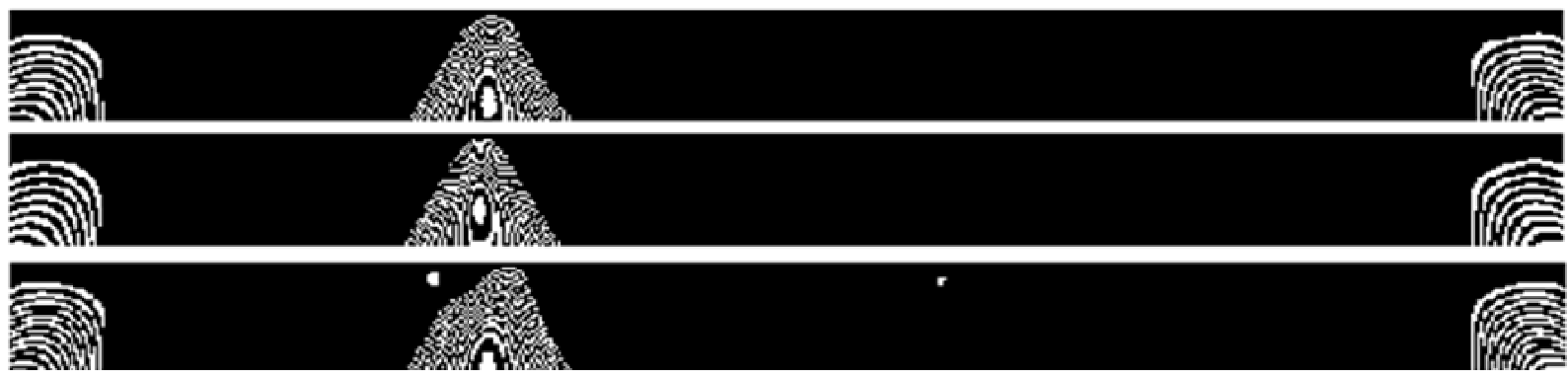

(a) Before flaw

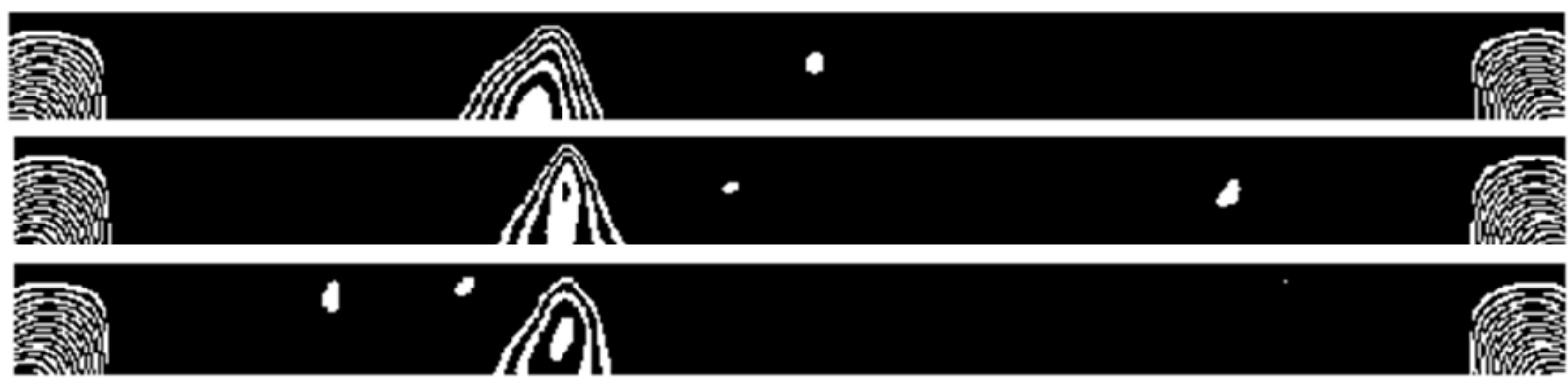

(b) Edge entering flaw

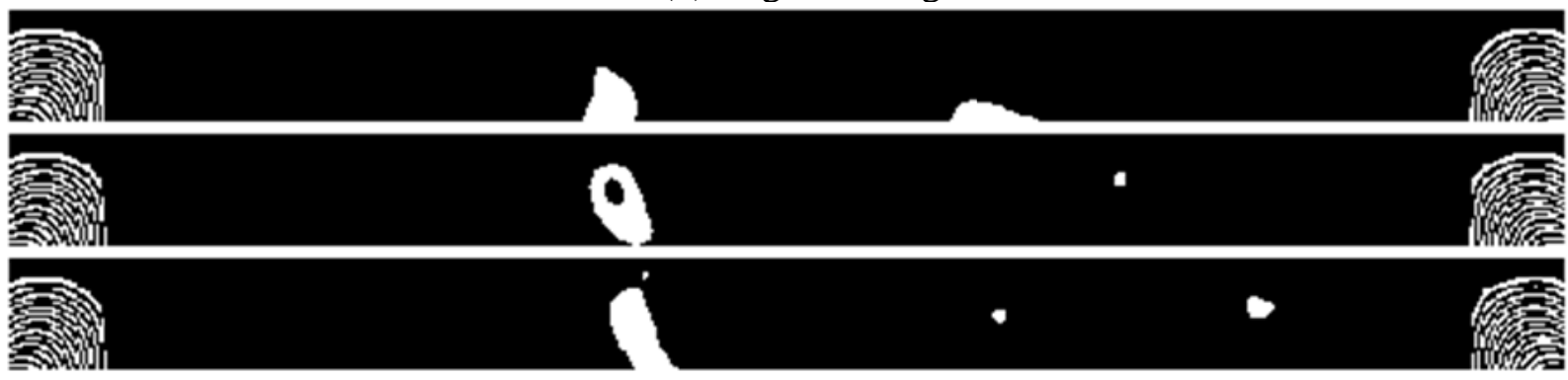

(c) Delamination

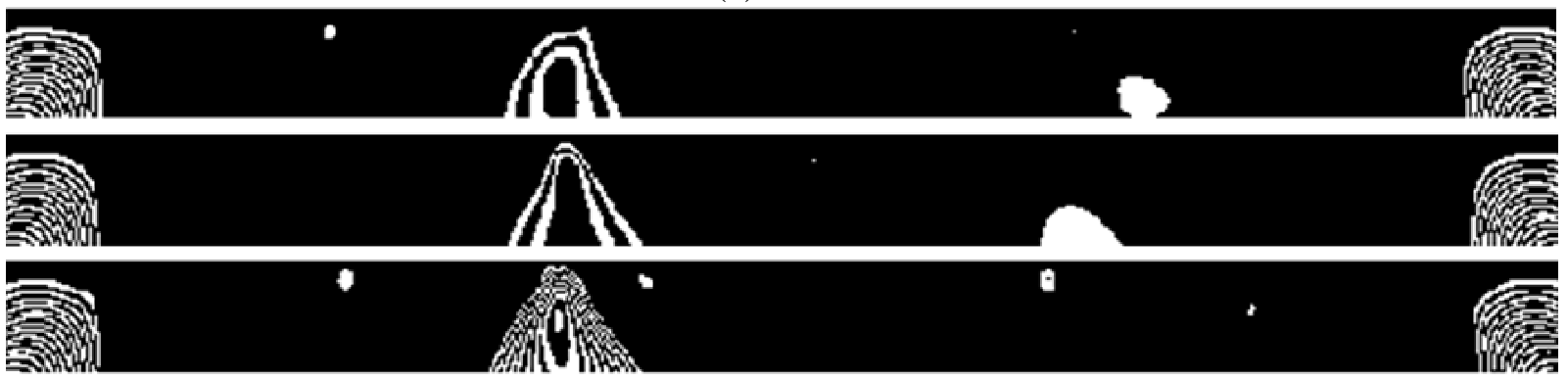

(d) Edge exiting flaw

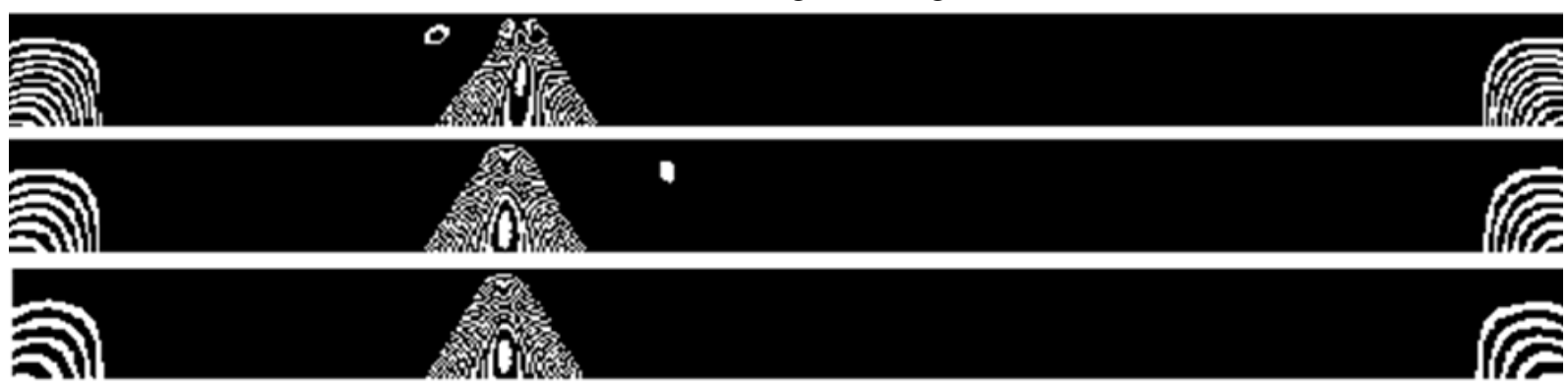

(e) After flaw

Fig. (7). Thumbprints for Portal $7200+$ Bitumastic 50 scan past delamination section. 
Turning our attention to the Protal $7200+$ Bitumastic sample we can see some of the fingerprint characteristics of both the HBE-95 and Bitumastic samples. As the parallel transducers move past the delaminated region (Fig. 7c), we see the effect of the loss of the epoxy thickness as the triangular feature moves to the right. Just as this mode was damped at the edge of the flaw for the Bitumastic 50 coating by itself we see the ridge count drop here as well. We also see the presence of a possible mode conversion since the delay of the guided wave mode when propagating directly through the flaw is so great. The amplitude here is once again drastically diminished from the signal with the unflawed coating. The similar results for the two coating sections with epoxy coatings indicate the viscoelastic material properties for these two epoxy layers are similar. There also seems to be a fairly large disconnect between the guided waves propagating directly through the epoxy delaminations. The manufactured delamination creates a step function in the thickness of the plate; this could result in a mode conversion to a much slower propagating mode. This could also be evidenced by edge effects of the delamination. The edge effects are present because of the large beam width of the guided waves. For these tests we used $500 \mathrm{kHz}, 1 "$ contact transducers. When more and more of the beam is propagating through the delamination, it affects more and more of the guided wave energy which translates to the gradual movement of the triangular feature to the right.

We are confident that we can identify the effects of a delamination under the epoxy coatings from the plate experiment. The question is what happens to the signals and DWFT features when we return to a real pipe sample. The ground flaw is only as deep as the thickness of the two protective coatings. The steel is very close to its original thickness. With the same parameters as the plate tests, we were able to record waveforms lengthwise down the pipe first in an area of the pipe surface that did not have any visible damage followed by propagation through the grounded area. This area has the same approximate size as the delaminations under the coatings on the plate test piece. (Fig. 8) shows the resulting thumbprints for the test propagation length of $431.8 \mathrm{~mm}$. Once again we see the shift to the right of the large triangular feature when the guided waves are propagating through the flaw. The more gradual thinning flaw shifts the arrival time, which coincides with the original notion that the guided waves in the coating-pipe system mimic those of the steel pipe itself. It is also noted that the plate coatings were made in our lab, so the thickness that we could uniformly achieve was $\sim 2 \mathrm{~mm}$, while the epoxy coating on the pipe sample is much thinner with the combined thickness of the epoxy and coal tar being $1 \mathrm{~mm}$. The difference in effective thickness loss accounts for the decrease in magnitude of the arrival shift.

\section{PIPELINE COATING SIMULATIONS}

EFIT is a full field numeric tool that can be used to visualize and follow what is happening to the elastic energy in a model. The interesting part about the pipeline coatings is that they are viscoelastic. These materials tend to have lower densities and slower shear wave velocities $\left(\mathrm{c}_{\mathrm{s}}\right)$. Since the cell size is determined by setting 10 grid points per slowest shear wavelength, including viscoelastic layers in the simulations increases the simulation space drastically. For the coal tar, Bitumastic 50 , the $\mathrm{c}_{\mathrm{s}}=750 \mathrm{~m} / \mathrm{s}$ which gives a cells size $\Delta \mathrm{s}=$ $7.4 \mathrm{e}^{-5} \mathrm{~m}$. In order to create a simulation space of $2^{\prime} \times 1.5^{\prime}$ which is on the order of the same dimensions as experiment, requires $6.91 \times 10^{9}$ cells. In order to perform the EFIT simulation this means that we need around 552.9 GB of physical memory. We accomplished this by using 260 cores for the parallel processing. With this huge simulation space, the cluster completed 83336 time steps in just over 98 hours.

We completed EFIT simulations for 2 different epoxy coatings as well, although they are slightly different materials than the experimental epoxies since all the needed viscoelastic properties are unavailable. Table $\mathbf{3}$ lists the material densities and wave speeds for all three of the coatings simulated.

Table 3. Material Properties for the Three Coatings as Well as that of the Steel Plate Substrate Used in the 3D EFIT Simulations

\begin{tabular}{|l|c|c|c|}
\hline \multicolumn{1}{|c|}{ Material } & $\boldsymbol{\rho}\left(\mathbf{k g} / \mathbf{m}^{\mathbf{3}}\right)$ & $\mathbf{V}_{\mathbf{l}}(\mathbf{m} / \mathbf{s})$ & $\mathbf{V}_{\mathbf{s}}(\mathbf{m} / \mathbf{s})$ \\
\hline \hline Steel & 7800 & 5940 & 3220 \\
\hline Carboline Bitumastic 50 & 1500 & 1860 & 750 \\
\hline Mereco 303 (Epoxy 1) [48] & 1080 & 2390 & 990 \\
\hline DE \& C 2057 / Ca9 (Epoxy 2) & 1600 & 2960 & 1450 \\
\hline
\end{tabular}

Fortunately, as expected from experiment, the shear wave velocities for both of the epoxies were greater than that of the Bitumastic 50, so we could more easily manage the use of the available computational cluster resources. This also results in shorter simulation times. We did run into some

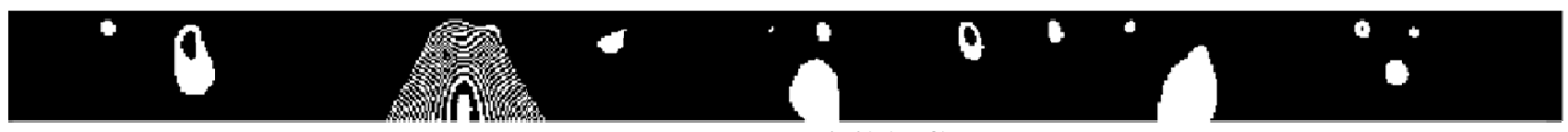

(a) No visible flaw

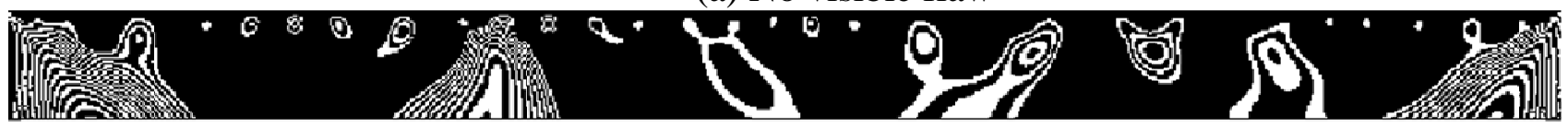

(b) Propagating through ground flaw

Fig. (8). Thumbprints derived from lengthwise experimental test of the DOT pipe sample. 
computer availability issues since these simulations require almost the entire parallel cluster at William and Mary.

The preliminary results from these simulations look promising and point toward more research to study the guided waves in multi-layered systems. From the raw waveforms (Fig. 9), we can see that there is some difference in the signals from experiment (Fig. 3). In the EFIT waveforms we can relate the structures seen in the plain steel simulation to the guided wave modes expected from the dispersion curves (Fig. 1). The waveforms for both the plain steel plate and the Bitumastic 50 coating are cut short because of computational cluster availability. Since the overall structure of the waveforms is so different from their experimental counterparts, the previously developed DWFT feature extraction algorithm does not produce meaningful results for these waveforms. Preliminary efforts show some interesting detail of some DWFT features. Fig. (10) shows an example of this line of investigation. It appears that the DFWT results in some fairly distinct features for each of the first three mode arrivals. The arrival of the later S0 mode isn't included because in order to have a wavelet thumb print window that was comparable across all of the EFIT signals we had to reduce its length. The blue brackets indicate the features corresponding to the S1 mode, the green ovals to the $\mathrm{A} 0$ and the red box to the A1. For the A0 and A1 modes, even though a shift in arrival is readily apparent, it seems there is a correlation to their size and shape to the material properties of the coating.

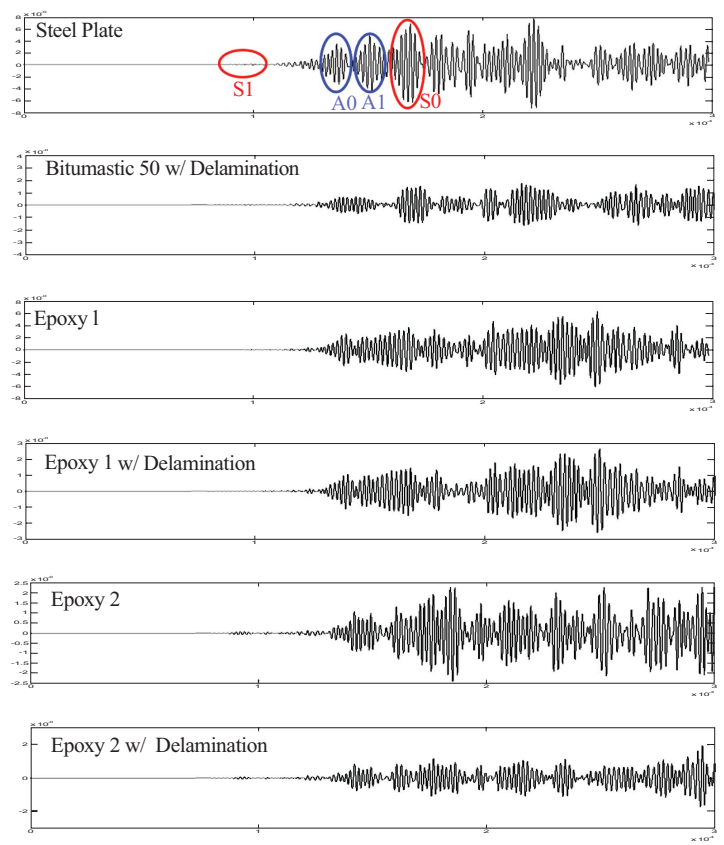

Fig. (9). The EFIT simulation results appear different from their experimental counterparts. In the steel plate waveforms we can see the structure of each of the guided wave modes expected frm the dispersion curves.

\section{CONCLUSIONS AND FUTURE WORK}

We have shown how elastic guided waves are a good tool for inspecting coated pipes for quantitative information about their structural status. Knowledge of how elastic guided waves behave for idealized cases allows for initial investigation of real structures. Full understanding of a system is derived from combining full field computational results with expertise from experimental aspects of an application. Even though we have presented work related to a specific structural health application, the same approach may be used for numerous other SHM applications involving plate-like and pipe-like structures. This is because we approach the problem from the point of extracting the meaningful guided wave information not the application itself. Once we have the waveform data, the extraction of the guided wave mode information is quite similar for a diverse range of physical situations. The meaning of this information is where the specific differences arise. We have shown results from interpreting guided wave interactions with delaminations of material layers with unknown material parameters that utilizes the dispersive nature of the guided wave modes to provide information about the composite structure. Since full analytic solutions for the real structures with flaws are not even conceptually possible, full field computer simulations using 3D EFIT are used to help interpret the signals from experiments and give us better insight in to what is happening to the guided wave energy as it interacts with various flaws.

It was demonstrated that the DWFT algorithm could successfully be used to show that differences can be identified between different unknown coatings on pipes. Furthermore, it was shown that the guided waves in layered material are sensitive to delaminations of the coating from the pipe wall. Because of the dispersive nature of the guided waves, they would change propagation velocity or mode convert depending on if the propagation path was directly through the delamination or not. We found that wavelet filtering with the DWFT could extract the feature corresponding to this change in guided wave propagation and render it as a change in the thumb print feature which is automatically interpretable as a change in the properties of the pipe and coating structure. The motivation was to be able to identify multiple types of coatings, with our preliminary findings from implementation of the EFIT simulation it appears that the DWFT features correspond in shape and size to the material properties of the coating as well as holding information of the arrival of the guided wave modes.

Further research is, of course, needed to systematically examine placement and sizing of flaws under many kinds of coatings in order to optimize the most informative feature extraction. Utilizing the EFIT simulations to examine what is happening in these signals would help identify the aspects of the experimental approach that are different from these. The signals are not so different that the underlying physics is being misrepresented in either case, but rather there are experimental effects that are that are hiding useful information in the noisy experimental data. The EFIT simulations could shed some light on how to either recreate the experimental data or how to process the data in a more informative way that also is upheld by the simulated data. Experimental samples could always have more flaws present than intended, but when trying to develop a system for inspection, it is important to fully understand the model 


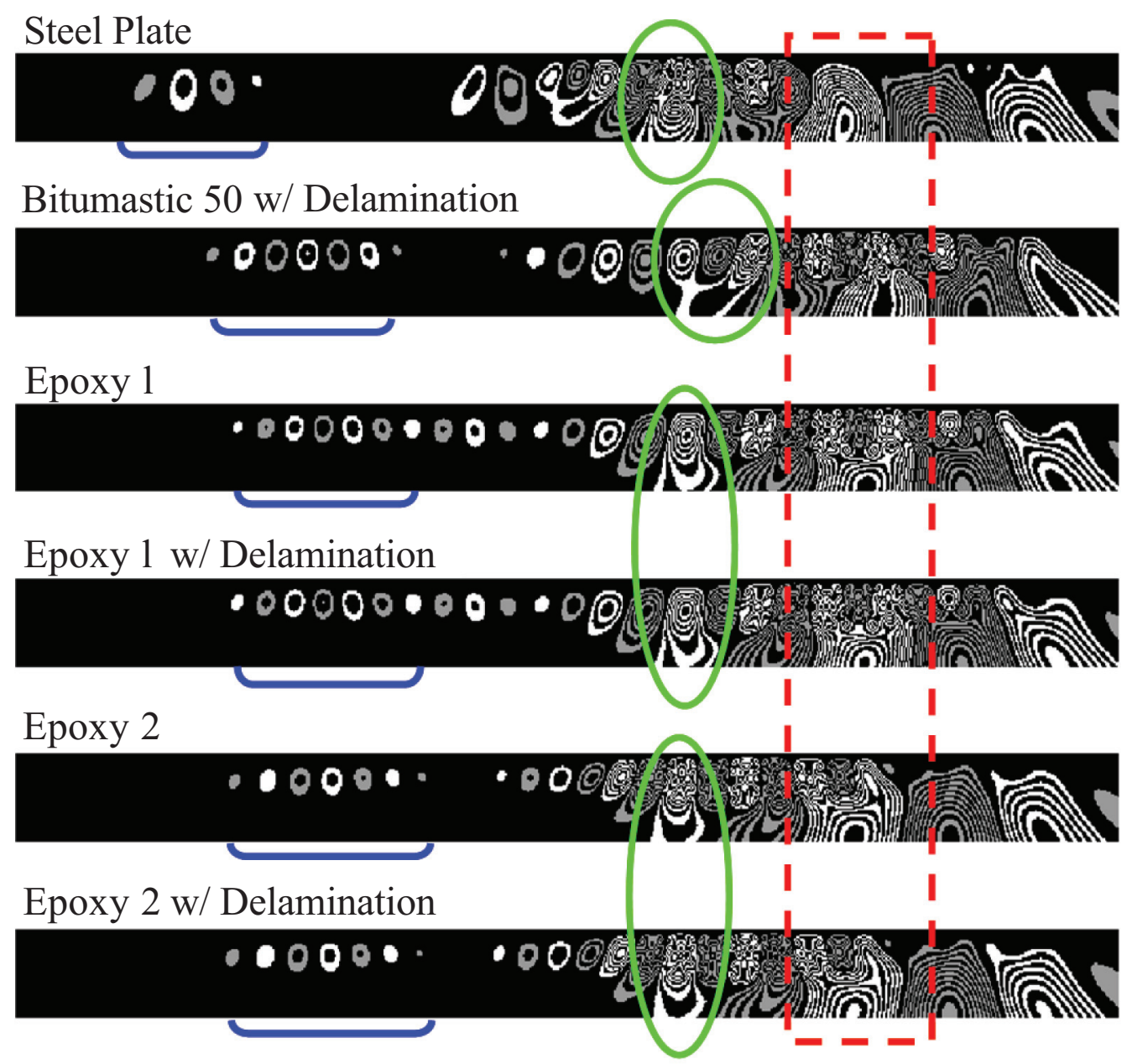

Fig. (10). The EFIT simulation viscoelastic coatings on a steel plate with a delamination produce some interesting features in the DWFT thumbprints. The blue brackets, green ovals and red box indicate features from the first three modes.

being worked with. Computer simulations allow for just this case, and although it is idealized, we can always step back to experiment for bench marking. The simulation space allows the researcher needed control over flaw dimensions and placement with respect to the transducers for the development of a robust automated flaw detection system.

\section{ACKNOWLEDGEMENTS}

The authors would like to thank Dr. Kevin Leonard for helpful discussions in the course of this work, which was partially supported by the Department of Transportation under contract \# DTRT57-07-C-10006. Thanks also to Tom Crockett for arranging to make the entire SciClone cluster available for the soft-coating simulations.

\section{REFERENCES}

[1] Achenbach J. Wave Propagation in Elastic Solids. Noth Holland, New York 1984.

[2] Auld BA. Acoustic Fields and Waves in Solids. 2nd ed. Malabar, FL: Kreiger Publishing 1990.

[3] Graff K. Wave motion in elastic solids. New York: Dover 1991.

[4] Rose J. Ultrasonic waves in solid media. New York: Cambridge Uniersity Press 1999.

[5] Viktorov I. Rayleigh and lamb waves - physical theory and applications. New York, NY: Plenum Press 1967.

[6] Raghavan A, Cesnik C. Review of guided-wave structural health monitoring. Shock Vib Digest 2007; 91-114.
[7] Rose J. A baseline and vision of ultrasonic guided wave inspection potential. J Pressure Vessel Technol 2002; 124: 273-82.

[8] Hinders M, Bingham J, Rudd K, Jones R, Leonard K. Wavelet thumbprint analysis of time domain reflecometry signals for wiring flaw detection. Rev Prog Quant Nondestruct Evaluat 2006; 25: 641-8.

[9] Hou J, Leonard K R, Hinders M K. Multi-mode lamb wave arrival time extraction of improved tomographic reconstruction. Rev Prog Quant Nondestruct Evaluat 2005; 24: 736-43.

[10] Leonard K, Hinders M. Lamb wave tomography of pike-like structures. Ultrasonics 2005; 43 (7): 574-83.

[11] Leonard K. Ultrasonic guided wave tomography of pipes. PHD Thesis, The College of William and Mary, Williamsburg 2004.

[12] Leonard K, Hinders M. Guided wave helical ultrasonic tomogaphy of pipes. J Acoust Soc Am 2003; 114 (2): 767-74.

[13] Leonard K, Hinders M. Lamb wave tomography of pipes and tanks using frequency compounding. Rev Prog Quant Nondestruct Evaluat 2005; 24: 867-74.

[14] Leonard K, Hinders M. Lamb waves helical ultrasonic tomography of pipes. Rev Prog Quant Nondestruct Evaluat 2004; 23: 173-9.

[15] Leonard K, Hinders M. Multi-mode lamb wave tomography with arrival time sorting. J Acoust Soc Am 2005; 117(4): 2028-38.

[16] Leonard K, Malyarenko E, Hinders M. Ultrasonic lamb wave tomography. Inverse Problems 2002; 18(6): 1795-808.

[17] Malyarenko E. Lamb wave diffraction tomography. PhD Thesis, The College of William and Mar, Williamsburg 2000.

[18] Malyarenko E, Hinders M. Fan beam and double crosshole lamb wave tomography for mapping flaws in aging aircraft structures. J Acoust Soc Am 2000; 108(4): 1631-9.

[19] Malyrenko E, Hinders M. Ultrasonic lamb wave diffraction tomography. Ultrasonics 2001; 39(4): 269-81. 
[20] McKeon J. Tomography applied to lamb wave contact scanning. PhD Thesis, The College of William and Mary, Williamsburg 1998.

[21] McKeon J, Hinders M. Lamb wave contact scanning tomography. Rev Prog Quant Nondestruct Evaluat 1999; 18: 951-8.

[22] McKeon J, Hinders M. Lamb wave scattering from a through hole. J Sound Vib 1999; 224 (5): 843-62.

[23] McKeon J, Hinders M. Parallel projection and crosshole lamb wave contact scanning tomography. J Acoust Soc Am 1999; 106(5): 2568-77.

[24] Rayleigh L. On Waves propagated along the plane surface of an elastic solid. Proc Lond Math Soc 1885; 17: 4-11.

[25] Lamb H. On waves in an elastic plate. Proc R Soc Lond Ser A 1917; XCIII: 114-28.

[26] Mindlin R. Influence of rotatory inertia and shear on flexural motions of isotropic elastic plates. J Appl Mech 1951;31-38.

[27] Worlton D. Ultrasonic testing with Lamb waves. Nondestruct Test 1957; 158 (4): 218-222.

[28] Worlton D. Experimental confirmation of Lamb waves at megacycle frequencies. J Appl Phys 1961; 32 (6): 967-71.

[29] Gazis D. Three-dimensional investigation of the propagation of waves in hollow cylinders i: analytical foundation. J Acoust Soc Am 1959; 31: 568-73.

[30] Gazis D. Three-dimensional investigation of the propagation of waves in hollow cylinders. ii: numerical results. J Acoust Soc Am 1959; 31: 573-8.

[31] Li J, Rose J. Natural beam focusing on non-axisymmetric guided waves in large-diameter pipes. Ultrasonics 2006; 44 (1): 35-45.

[32] Luo W, Zhao X, Rose J. Guided wave plate experimet for a pipe. J Pressure Vessel Technol 2005; 127: 345-50.

[33] Nayfeh A, Nassar E. Simulation of the influence of bonding materials on the dynamic behavior of laminated composites. J Appl Mechan 1978; 45: 822-8.

[34] Claus R, Kline R. Adhesive bondline interrogation using stoneley wave methods. J Appl Phys 1979; 50 (12): 8066-9.

[35] Schoenberg M. Elastic wave behavior across linear slip interfaces. J Acoust Soc Am 1980; 68 (5): 1516-21.

[36] Tsukahara Y, Ohira K. Detection of smooth bondings of polymer coating by ultrasonic spectroscopy. Ultrasonics 1989; 27: 3-7.

[37] Xu P, Datta S. Guided waves in a bonded plate: aparametric study. J Appl Phys 1990; 67 (11): 6779-86.

[38] Nayfeh A. The general problem of elastic wave propagation in multilayered anisotropic media. J Acoust Soc Am 1991; 89(4): 1521-1531.

[39] Laperre J, Thys W. Experimental and theoretical study of lamb wave dispersion in aluminum/polymer bilayers. J Acoust Soc Am 1993; 94 (1): 268-78.

[40] Xu P, Lindenschmidt K, Meguid S. A new high-frequency analysis of coatings using leaky lamb waves. J Acoust Soc Am 1993; 94 (5): 2954-62.

[41] Rose J, Pilarski A, Rajana K, Ditri J. Coating influence on generation and reception of ultrasonic lamb type plate waves. Rev Prog Quant Nondestruct Evaluat 1994; 13: 1903-1910

[42] Lowe M. Matrix techniqes for modeling ultrasonic waves in multilayered media. IEEE Trans Ultrson Ferroelectr Freq Control 1995; 42 (4): 525-42.

[43] Moulin E, Assaad J, Delebarre C, Osmont D. Modeling of Lamb waves generated by integrated tranducers in composite plates using a coupled finite element-normal modes expansion method. J Acoust Soc Am 2000; 107 (1): 87-94.

[44] Moulin E, Assaad J, Delebarre C, Grondel S, Balageas D. Modeling of integrated lamb waves generation systems using a oupled finite element-normal modes expansion method. Ultrasonics 2000; 38: 522-6.
[45] Duquenne L, Moulin E, Assaad J, Grondel S. Transient modeling of Lamb waves generated in viscoelastic materials by surface bonded piezoelectric transducers. J Acoust Soc Am 2004; 116 (1): 133-41.

[46] Galan J, Abascal R. Remote characterization of defects in plates with viscoelastic coatings using guided waves. Ultrasonics 2004; 42: 877-82.

[47] Barshinger J, Rose J. Guided wave propagation in an elastic hollow cylinder coated with a viscoelastic material. IEEE Trans Ultrason Ferroelectr Freq Control 2004; 51 (11): 1547-56.

[48] Luo W, Rose J. Phased array focusing with guided waves in a viscoelastic coated hollow cylinder. J Acoust Soc Am 2007; 121 (4): 1945-55.

[49] Fellinger P, Marklein R, Langenberg K, Klaholz S. Numerical modeling of elastc wave propagation and scattering with EFITelastodynamic finite integration technique. Wave Motion 1995; 21 (1): 47-66.

[50] Schubert F, Peiffer A, Kohler B, Sanderson T. The elastodynamic finite integration technique for waves in cylindrical geometries. J Acoust Soc Am 1998; 104 (5): 2604-14.

[51] Schubert F, Koehler B. Three-dimensional time domain modeling of ulrasonic wave propagation in concrete in explicit consideration of aggregates and porosity. J Comput Acoust 2001; 9(4): 1543-60.

[52] Schubert F. Numerical time-domain modeling of linear and nonlinear ultrasonic wave propagation using finite integration techniques - theory and aplications. Ultrasonics 2004; 42: 221-9.

[53] Rudd K, Leonard K, Bingham J, Hinders M. Simulation of guided waves in complex piping geometries using the elastodynamic finite integratio technique. J Acoust Soc Am 2007; 121 (3): 1449-58.

[54] Abbate A, Koay J, Frankel J, Schroeder SC, Das P. Application of wavelet transform signal processor to ultrasoud. IEEE Ultrason Symp 1994; 1147-52.

[55] Masscotte D, Goyette J, Bose T. Wavelet-transorm-based method of analysis for lamb-wave ultrasoni nde signals. IEEE Trans Instrum Measure 2000; 49 (3): 524-9.

[56] Perov D, Rinkeich A, Smorodinskii Y. Wavelet filtering of signals for ultrasonic flaw detector. Russian J Nondestruct Test 2002; 38 (12): 869-82.

[57] Lou H, Hu G. An approach based on simplified klt and wavelet transform for enhaning speeh degraded by non-stationary wideband noise. J Sound Vib 2003; 268: 717-29.

[58] Zou J, Chen J. A comparative study on time-frequency frature of cracked rotor by wigner-ville distribution and wavelet transform. J Sound Vib 2004; 276: 1-11.

[59] Hou J. Ultrasonic signal detection and recognition using dynamic wavelet fingerprints. PhD Thesis, The College of William and Mary, Williamsburg 2004

[60] Hou J, Hinders M. Dynamic wavelet fingerprint identification of ultrasound signals. Mater Evaluat 2002; 60 (9): 1089-93.

[61] Hou J, Leonard K, Hinders M. Automatic multi-mode lamb wave arrival time extraction for improved tomographic reconstrucion. Inverse Problems 2004; 20 (6): 1873-88.

[62] Hinders M, Leonard K, Malyarenko E. Blind test of Lamb wave diffraction tomography. Rev Prog Quant Nondestruct Evaluat 2002; 21:278-83.

[63] Hinders M, Jones R, Leonard K, Rudd K. Wavelet thumbprint analysis of time domain reflectometry signals for wiring flaw detection. Eng Intelligent Syst Electric Eng Commun 2007; 15 (4): 225-39.

[64] Hou J, Rose S, Hinders M. Ultrasonic periodontal probing based on the dynamic wavelet fingerprint. Eur J Appl Signal Proces 2005; 7: $1137-46$. 